\title{
Non-Invasive Evaluation of Hepatic Fibrosis for Type C Chronic Hepatitis
}

\author{
Chie Tatsumi $^{\mathrm{a}}$ Masatoshi Kudo $^{\mathrm{a}}$ Kazuomi Ueshima ${ }^{\mathrm{a}}$ Satoshi Kitai ${ }^{\mathrm{a}}$ \\ Emi Ishikawa $^{a}$ Norihisa Yada $^{a}$ Satoru Hagiwara ${ }^{a}$ Tatsuo Inoue ${ }^{a}$ \\ Yasunori Minami $^{\mathrm{a}}$ Hobyung Chung $^{\mathrm{a}}$ Kiyoshi Maekawa $^{\mathrm{b}}$ Kenji Fujimoto $^{c}$ \\ Michio Kato ${ }^{c}$ Akiko Tonomurad Tsuyoshi Mitake ${ }^{d}$ Tsuyoshi Shiina ${ }^{e}$ \\ ${ }^{a}$ Department of Gastroenterology and Hepatology, Kinki University School of Medicine, and ${ }^{b}$ Division of \\ Abdominal Ultrasound, Department of Laboratory Medicine, Kinki University School of Medicine, Osaka-Sayama, \\ 'Department of Internal Medicine, National Hospital Organization Minami-Wakayama Medical Center, Tanabe, \\ ${ }^{\mathrm{d} H i t a c h i}$ Medical Corporation, Tokyo, and 'Human Health Science, Graduate School of Medicine, Kyoto University, \\ Kyoto, Japan
}

\section{Key Words}

Biopsy, liver · FibroScan · Fibrosis, liver - Real-time tissue elastography

\begin{abstract}
Objective: The aim of this study was to investigate liver fibrosis using non-invasive Real-time Tissue Elastography ${ }^{\circledR}$ (RTE) and transient elastography (FibroScan ${ }^{\circledR}$ ) methods. Methods: RTE, FibroScan and percutaneous liver biopsy were all performed on patients with chronic liver disease, particularly hepatitis C, to investigate liver fibrosis. Results: FibroScan and RTE were compared for fibrous liver staging (F stage), which was pathologically classified using liver biopsy. In FibroScan, significant differences were observed between F1/F3 and F2/F4, but no such differences were observed between F1/F2, F2/F3 and F3/F4. In RTE, significant differences were observed between F1/F2, F2/F3 and F2/F4. But for F3/F4, no significant differences were observed. Conclusion: FibroScan and RTE correlated well with F staging of the liver. In particular RTE was more successful than FibroScan in diagnosing the degree of liver fibrosis.
\end{abstract}

Copyright $\odot 2010$ S. Karger AG, Basel

\section{KARGER}

Fax +4161306 1234 E-Mail karger@karger.ch www.karger.com
(C) 2010 S. Karger AG, Basel

$0300-5526 / 10 / 0531-0076 \$ 26.00 / 0$

Accessible online at:

www.karger.com/int

\section{Introduction}

Currently, percutaneous liver biopsy is considered to be the gold standard for determining the index of the liver fibrosis in a patient with chronic liver disease including hepatitis type C. However, liver biopsy is associated with risks of complications, lacks accuracy due to sampling error, and is physically and psychologically uncomfortable for the patient. Transient elastography $\left(\right.$ FibroScan ${ }^{\circledR}$ ) and Real-Time Tissue Elastography ${ }^{\circledR}$ (RTE) have been developed as non-invasive methods to evaluate the degree of liver fibrosis, potentially providing alternatives to liver biopsy. FibroScan detects the propagation speed of a shear wave transmitted from a probe through the liver and calculates the shear modulus of the liver to evaluate the degree of liver fibrosis [1-3]. On the other hand, RTE visualizes a 2-dimensional strain image induced by external freehand compression with the probe or by internal heartbeats. To evaluate the degree of liver fibrosis, it is reported that the pattern of strain image induced by compression becomes patchy as fibrosis progresses [4]. To increase objectivity,

Masatoshi Kudo, MD, PhD, Division of Gastroenterology and Hepatology Department of Internal Medicine, Kinki University School of Medicine 377-2, Ohno-Higashi, Osaka-Sayama 589-8511 (Japan)

Tel. +81723660221 , ext. 3149, Fax +81 723672880

E-Mail m-kudo@med.kindai.ac.jp 
Fig. 1. Principle of elasticity imaging.
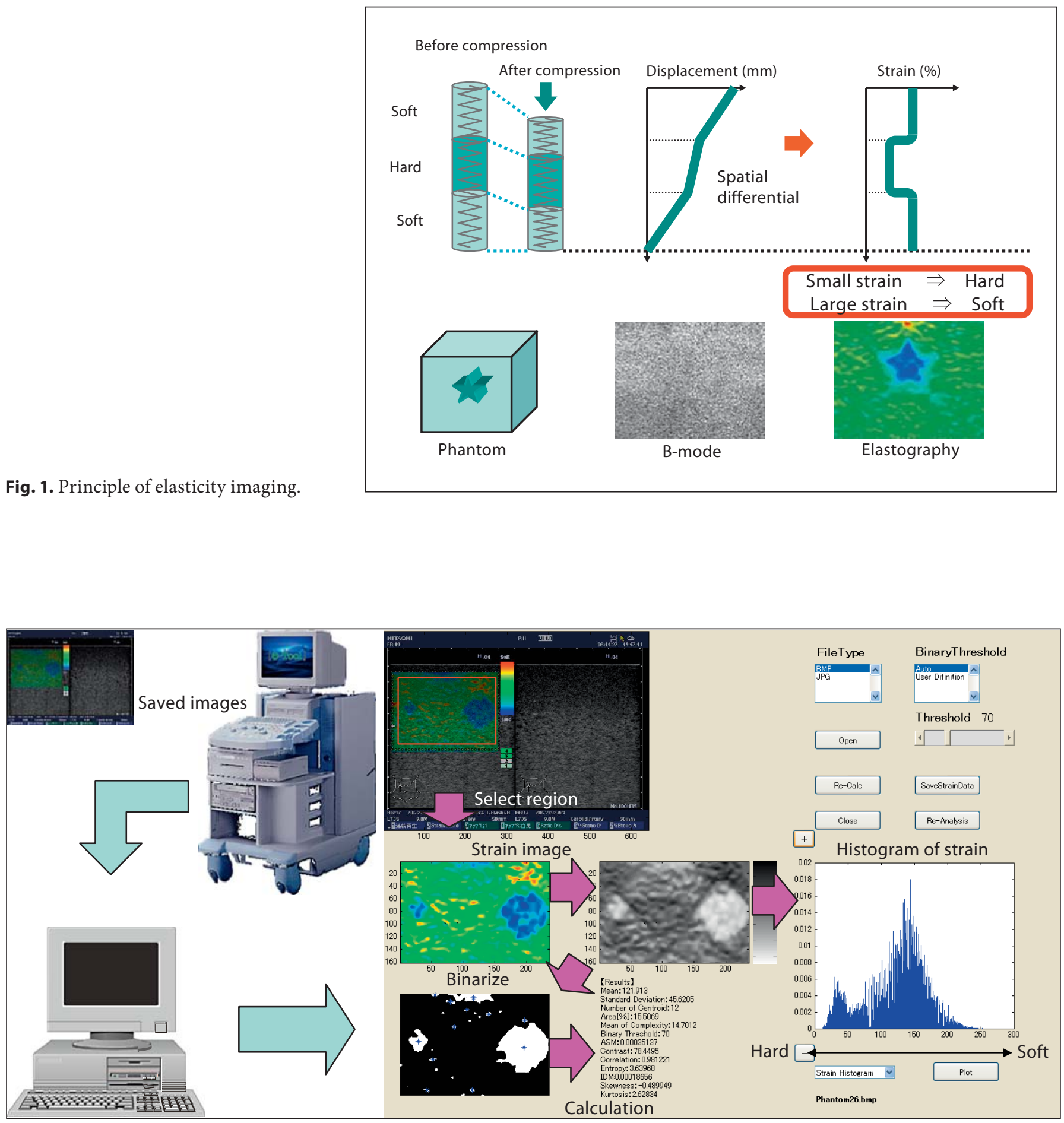

Fig. 2. Analysis tool. Mean = Mean of relative strain value; $\mathrm{SD}=$ standard deviation of relative strain value; area[\%] = ratio of blue area in the analyzed region; complexity = complexity of blue area [calculated as (boundary length) ${ }^{2} /$ area]. 
Fig. 3. Example of liver RTE image and result of liver fibrosis index (F stage 1).
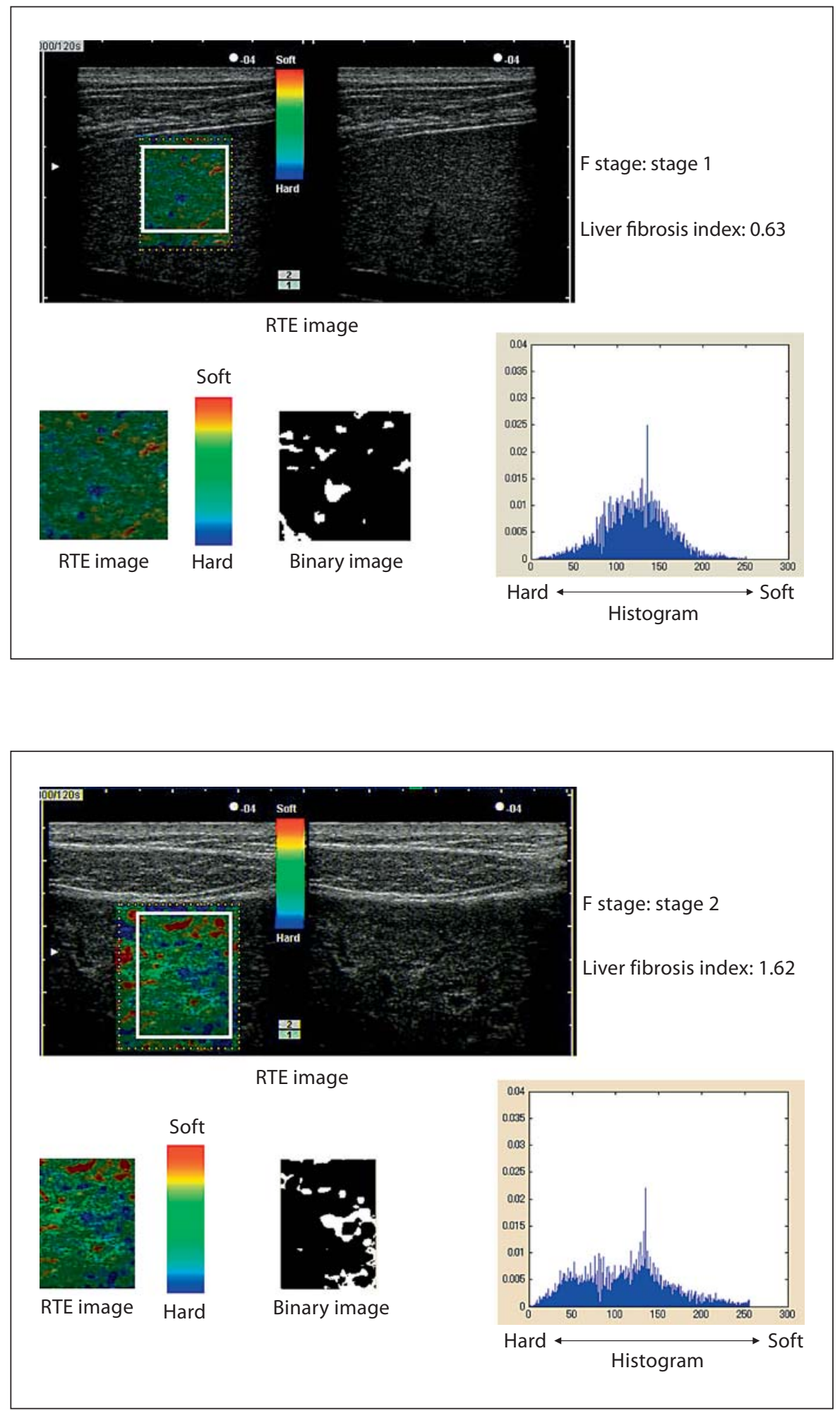

Fig. 4. Example of liver RTE image and result of liver fibrosis index (F stage 2). 
we proposed an image analysis method to evaluate strain image features [5]. In this paper, we propose a new algorithm for RTE to deliver an index which corresponds to the liver fibrosis stage (F stage), and report our clinical experience.

\section{Methods}

Before IFN treatment, percutaneous liver biopsy, FibroScan and RTE examinations were performed on 44 patients with chronic hepatitis $\mathrm{C}$. All patients gave their consent for this study. Of these 44 patients, 12 had F1, 9 had F2, 10 had F3, and 13 had F4, all diagnosed pathologically using percutaneous liver biopsy. FibroScan and RTE were performed before the liver biopsy for comparison with the F stage of the liver biopsy specimen. Measurements with FibroScan (EchoSens, Paris, France) were performed on the right lobe of the liver through intercostal spaces. The mean of 10 valid measurements was used as the index of liver stiffness. RTE was performed with EUB-8500 (Hitachi Medical Corp., Tokyo, Japan) through the right intercostal spaces to obtain the elastography images of the liver.

RTE displays elastic information of the tissue calculated from the tissue displacement. The tissue displacement is caused either by manual compression and relaxation of the probe or by the internal compression and relaxation with the heart. The principles underlying RTE are shown in figure 1 using a spring model with the hard and soft springs connected in series. When the spring is compressed, a hard spring is displaced to a lesser extent than a soft spring, thus the strain calculated from the displacement is small in hard springs and large in soft springs. RTE visualizes the relative strain using color gradations, similar to ultrasound color Doppler imaging. RTE is being clinically used and studied in various regions, such as breast, [6], thyroid gland, and prostate [7]. As discussed in the previous study, the RTE image shows a patchy pattern of colors as liver fibrosis progresses from hepatitis to cirrhosis $[4,5]$. This is because the fibrotic region is harder than the normal liver parenchyma and does not spread uniformly. For evaluation of liver, the strain induced by heartbeats (diastole) is used to perform RTE to reduce interobserver variability by compressing with an external probe. Six RTE images were collected for each patient and analyzed with the prototype analysis software shown in figure 2 to calculate 9 image features: mean of relative strain value; standard deviation of relative strain value; ratio of blue area in the analyzed region; complexity of blue area; kurtosis of strain histogram; skewness of strain histogram; entropy; inverse difference moment, and angular second moment. Multiple regression analysis was then performed with these 9 image features to quantify the index of liver fibrosis. Examples of liver RTE images and index of liver fibrosis results are shown in figures 3-6. As can be seen from these figures, as F stage progresses, the liver fibrosis index increases.

\section{Results}

FibroScan and RTE findings were compared against pathologically classified F-staged patients using liver biopsy. In FibroScan, significant differences were observed between F1/F3 and F2/F4, but no significant differences were observed between F1/F2, F2/F4 and F3/F4 (fig. 7).

In RTE, significant differences were observed between F1/F2, F2/F3 and F2/F3, but no significant differences were recognized between F3/F4 (fig. 8).

\section{Discussion}

The percutaneous liver biopsy is most reliable but it is invasive and cannot be performed frequently to study the progress of fibrosis. Thus non-invasive techniques, such as FibroScan and RTE, are more desirable.

FibroScan is simple and easy to use and displays the results on a monitor immediately. The result is based on 1-dimensional information (1-line) only. In the case of RTE, it visualizes 2-dimensional elastic information in real time and can be used in most patients.

FibroScan has many limitations. It cannot be used in patients with ascites, thick subcutaneous fat, narrow intercostal spaces, and hepatic atrophy. RTE does not have such limitations and it can be used in almost all patients, including those with the conditions mentioned above. However, RTE requires training to scan patients to obtain reproducible images and to analyze the data. To address these issues in RTE, we are investigating easy-to-use acquisition techniques to reduce interobserver variability and also to simplify RTE image acquisition.

FibroScan and RTE both correlate highly with the F staging of the liver using biopsy. In particular RTE is very useful for the differential diagnosis and staging of the liver fibrosis.

In future, we plan to study more cases to evaluate $\mathrm{Fi}$ broScan and RTE for liver fibrosis staging and to establish guidelines to minimize unnecessary liver biopsies, which will significantly benefit patients with chronic liver diseases.

\section{Disclosure Statement}

The authors declare that they have no financial conflict of interest. 
Fig. 5. Example of liver RTE image and result of liver fibrosis index (F stage 3 ).

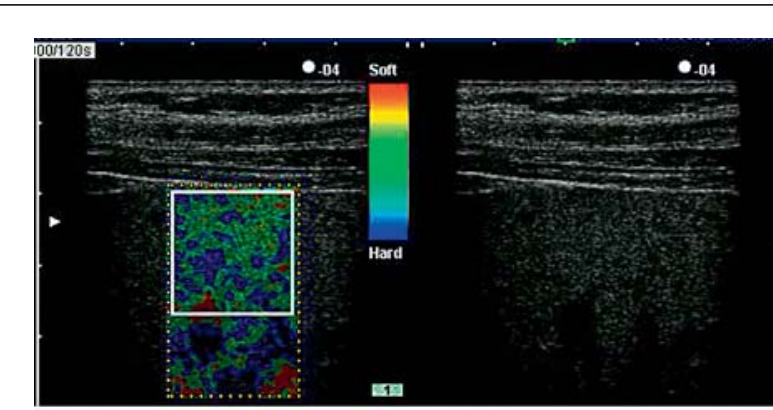

F stage: stage 3

Liver fibrosis index: 2.96

RTE image

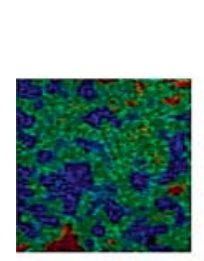

RTE image
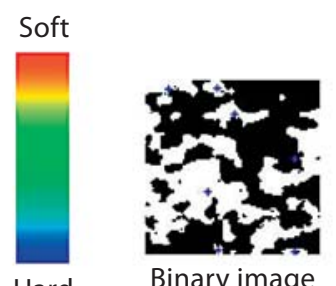

Binary image
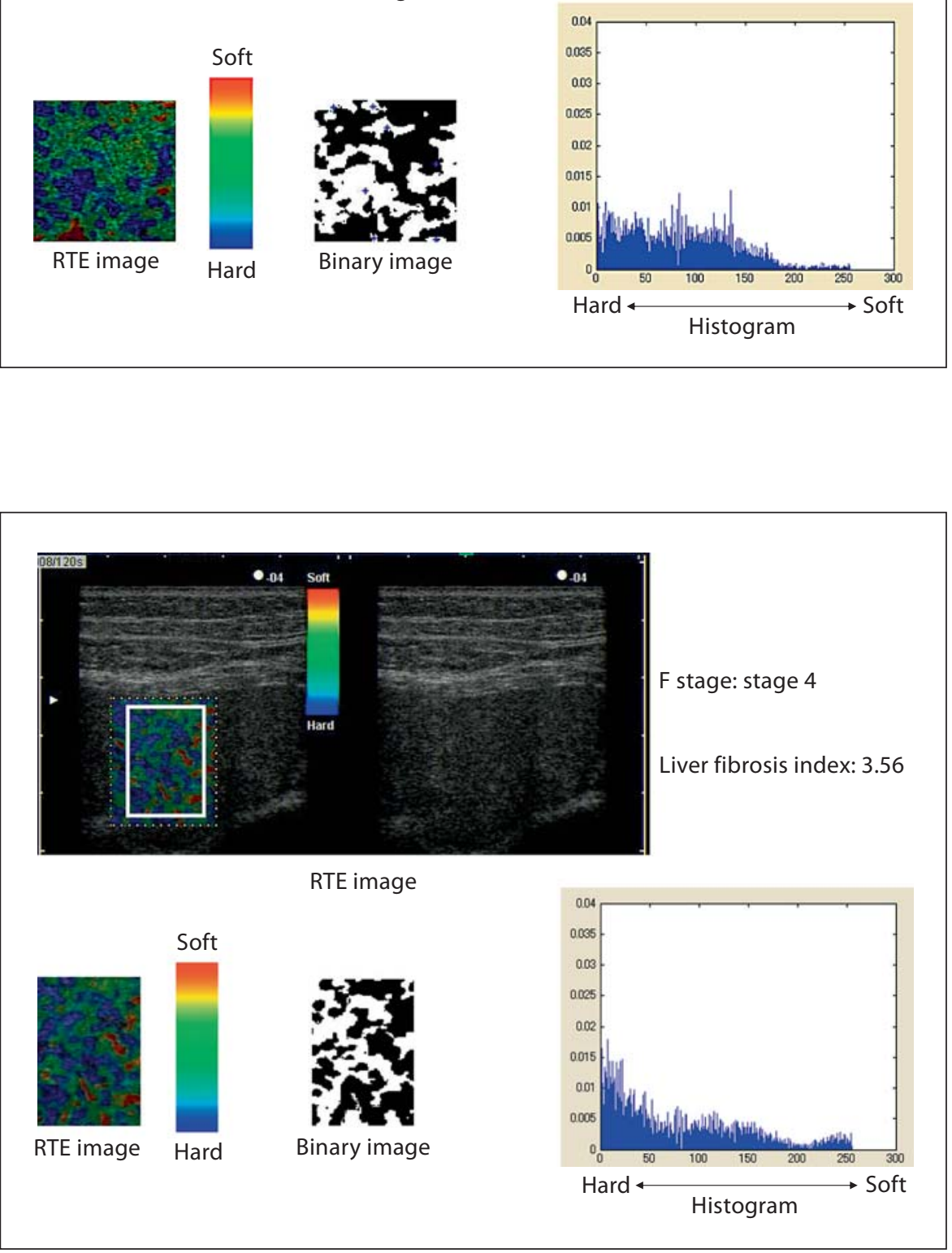

Fig. 6. Example of liver RTE image and result of liver fibrosis index (F stage 4). 


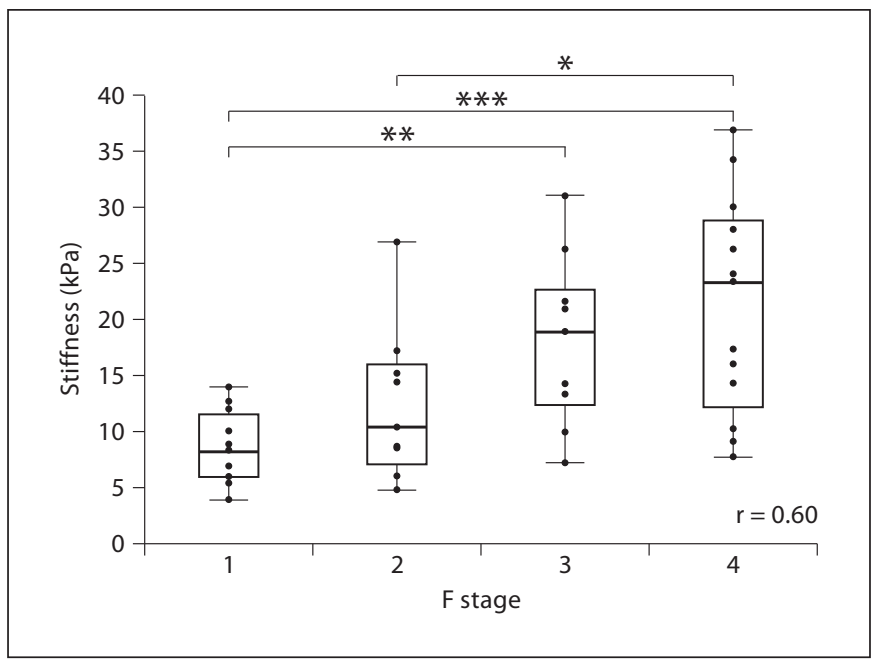

Fig. 7. FibroScan and F stage. ${ }^{*} \mathrm{p}<0.05 ;{ }^{* *} \mathrm{p}<0.005$; ${ }^{* *} \mathrm{p}<$ 0.001 .

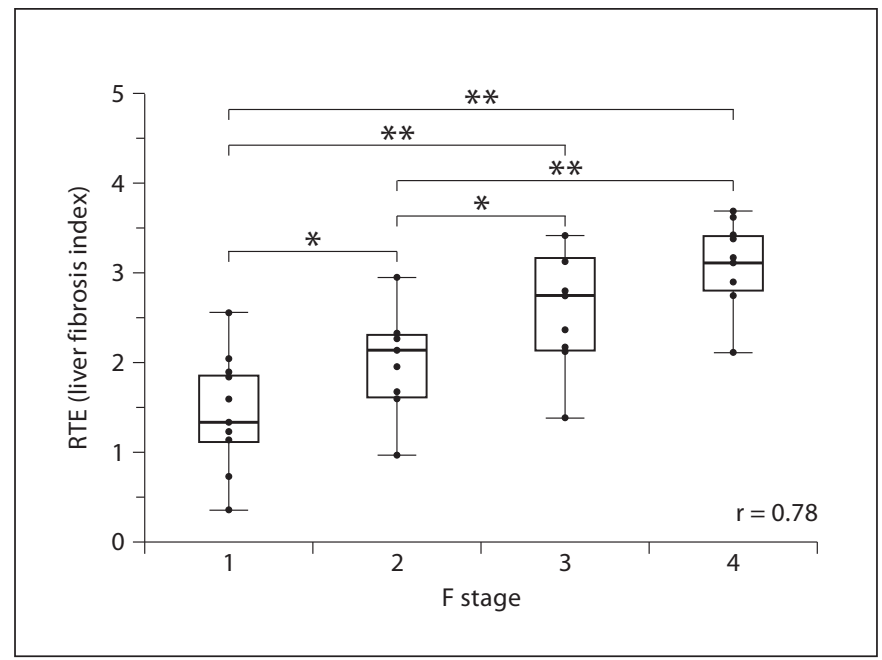

Fig. 8. RTE (liver fibrosis index) and F stage. ${ }^{*} \mathrm{p}<0.05$; ${ }^{* *} \mathrm{p}<$ 0.001 .

\section{References}

1 Sandrin L, Fourquet B, Hasquenoph JM, et al: Transient elastography: a new noninvasive method for assessment of hepatic fibrosis. Ultrasound Med Biol 2003;29:17051713.

2 Foucher J, Chanteloup E, Vergniol J, et al: Diagnosis of cirrhosis by transient elastography (FibroScan): a prospective study. Gut 2006;55:403-408.
3 Fraquelli M, Rigamonti C, Casazza G, et al: Reproducibility of transient elastography in the evaluation of liver fibrosis in patients with chronic liver disease. Gut 2007;56:968973.

4 Fujimoto K, Wada S, Oshita M, et al: Noninvasive evaluation of hepatic fibrosis in $\mathrm{pa}-$ tients with chronic hepatitis $\mathrm{C}$ using elastography. Medix Suppl 2007;24-27.

5 Tatsumi C, Kudo M, Ueshima K, et al: Noninvasive evaluation of hepatic fibrosis using serum fibrotic markers, transient elastography (FibroScan) and real-time tissue elastography. Intervirology 2008;51(suppl 1):27-33.
6 Itoh A, Ueno E, Tohno E, et al: Breast disease: clinical application of US elastography for diagnosis. Radiology 2006;239:341-350.

7 Tsutsumi M, Miyagawa T, Matsumura T, et al: The impact of real-time tissue elasticity imaging (elastography) on the detection of prostate cancer: clinicopathological analysis. Int J Clin Oncol 2007;12:250-255. 